\title{
Evaluation method of image resolution for the aberration-corrected STEM
}

Yasuhiko Sugigaki, Yoshifumi Taniguchi, Yudai Kubo, Kuniyasu Nakamura, Susumu Koyama and Mitsugu Sato

Hitachi High-Tech Corporation, Hitachinaka-shi, Ibaraki, Japan

The progress of aberration-corrected scanning transmission electron microscope (STEM) enables us to directly acquire images containing individual atoms in a crystallinity specimen. However, the atomic resolution of aberration-corrected STEM is usually defined by the atomic distance of dumbbell structure, and then, it is determined as a discrete value of the lattice spacing of the dedicated specimen rather than a continuous value. Sometimes such dedicated specimen is difficult to prepare and require the operator a skill of high level with specimen orientation. This paper proposes a new algorithm for evaluating atomic resolution on the basis of Rayleigh's criterion, which enables a continuous value of atomic resolution. Figure 1 shows a dark-field STEM image of Si[110] obtained by using the Hitachi HF5000, an aberration corrected TEM/STEM, and pixel intensity profile of the dumbbell image. In this algorithm, image orientation and unevenness of image intensity are compensated for in advance. After those image processing, hundreds of atomic dumbbells recorded in the STEM image are accumulated, and a very low noise dumbbell image is produced for accurate calculation. Figure 1 also shows the accumulated dumbbell image and its pixel intensity profile. As showing in Figure 1, Gaussian distribution can well fit to the accumulated dumbbell profile and separate into two Gaussian distributions. Then the standard deviation $(\sigma)$ of Gaussian distribution is evaluated from the accumulated dumbbell profile.

Figure 2 shows the definition of Rayleigh criterion which is applied to Gaussian distribution. The Rayleigh resolution "Res" based on Rayleigh criterion is given by

Res $=2 \sqrt{ } 2 \sigma$

Here $\sigma$ is standard deviation which is obtained by fitting the Gaussian distribution to the dumbbell profile. The dumbbell image $\mathrm{I}(\mathrm{x}, \mathrm{y})$ in dark-field STEM is represented by [1][2]

$\mathrm{I}(\mathrm{x}, \mathrm{y})=\sigma \mathrm{m} 2 \mathrm{Vp} 2(\mathrm{x}, \mathrm{y}) \otimes \operatorname{Jprob}(\mathrm{x}, \mathrm{y})$

Where $\sigma \mathrm{m}$ is the interaction constant, $\mathrm{Vp}$ is the projected potential with a single atom of the specimen and Jprob is the beam intensity profile. We assume that Jprobe is Gaussian distribution. The operator " $\bigotimes$ " denotes the convolution. From Equation (2), we can evaluate beam size from Rayleigh resolution "Res" for the dumbbell profile and the projected potential Vp.

By using this algorithm, the Rayleigh resolution of the STEM image shown in Figure 2 was calculated as $107 \mathrm{pm}$ and the effective beam size was calculated as $83 \mathrm{pm}$. Thus, the atomic resolution of aberration corrected STEM is evaluated as a continuous value. 
DF-STEM image

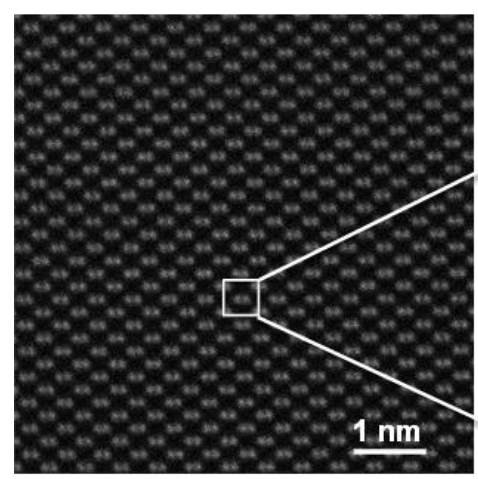

○ Pixel intensity

- Gaussian distribution (STD Dev. $\sigma=37.8 \mathrm{pm}$ )

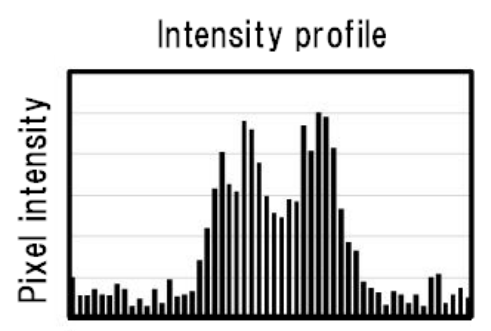

Dumbbell profile
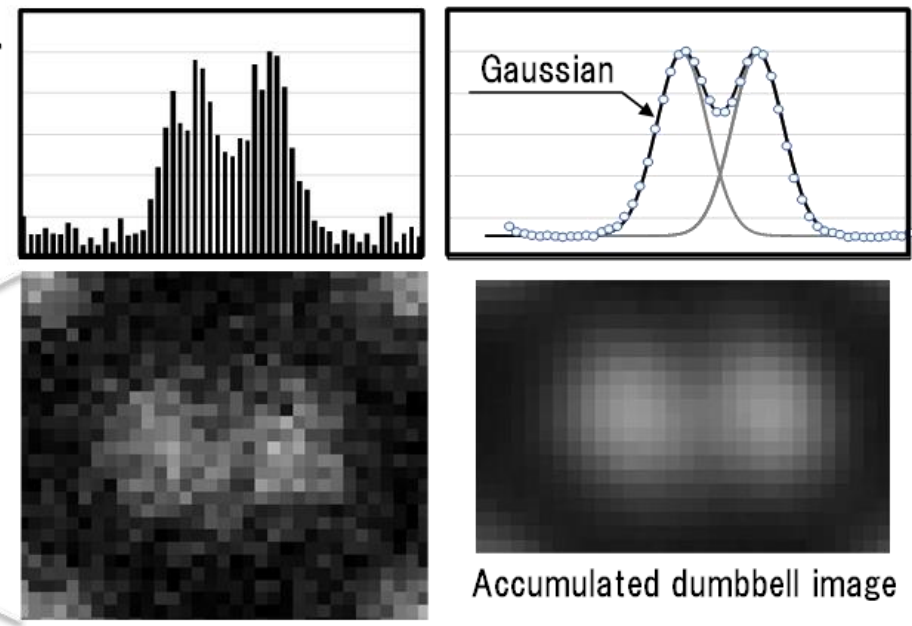

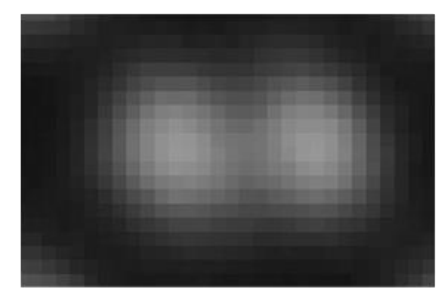

Accumulated dumbbell image

Figure 1. DF-STEM image of Si[110] obtained by using the Hitachi HF5000, an aberration corrected TEM/STEM, and the pixel intensity profile of dumbbell image.

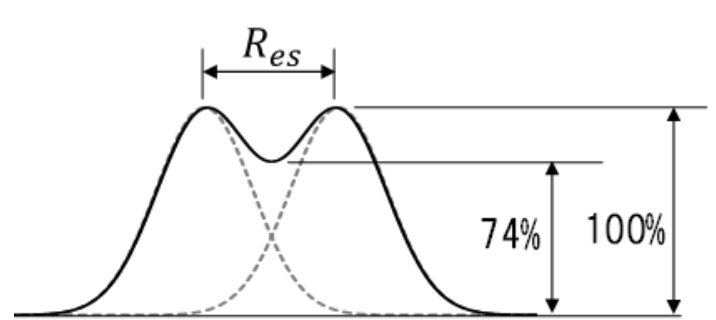

Rayleigh criterion for Gaussian distribution

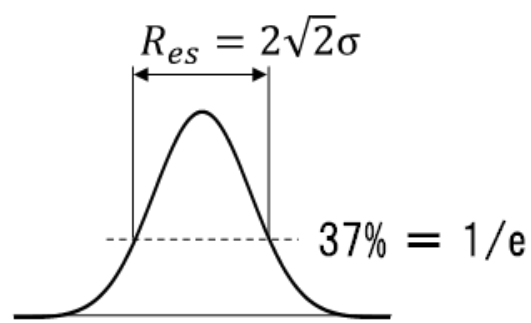

Gaussian distribution

( $\sigma$ : standard deviation)

Figure 2. Rayleigh criterion applied to the Gaussian distribution.

\section{References}

[1] D.E.Jesson and S.J.Pennycook: Proc. Roy. Soc. Lond., A441, 26 (1993).

[2] K. Nakamura, H. Kakibayashi, K. Kanehori and N. Tanaka, Journal of Electron Microscopy 1, 33 (1997). 